\title{
Heterogeneous-surface-mediated crystallization control
}

\author{
Shifeng Zhou ${ }^{1}$, Binbin Zheng ${ }^{2}$, Yasuhiko Shimotsuma ${ }^{3}$, Yunhao $\mathrm{Lu}^{2}$, Qiangbing Guo ${ }^{2}$, Masayuki Nishi ${ }^{3}$, \\ Masahiro Shimizu ${ }^{3}$, Kiyotaka Miura ${ }^{3}$, Kazuyuki Hirao ${ }^{3}$ and Jianrong Qiu ${ }^{1}$
}

The crystallization of matter at interfaces has long been a significant issue in science and technology, but surface-directed crystallization with controlled kinetics remains a matter of challenge. Here, we demonstrate a conceptual novel mechanism to steer liquid-solid phase transformation at interfaces by tailoring the chemical and structural inhomogeneity of a glass substrate through self-limited nanocrystallization of the glassy phase. Importantly, this approach enables large-scale development of metastable crystallization products, such as nanowire membranes. The thorough studies of the intermediate stages of crystallization reveal a unique cooperative mechanism in which the intricate interplays between inherent nanoscale forces and unique heterogeneous surfaces contribute to the mesoscale structural transformation from isolated units to superstructures. We further show that the constructed superstructures offer unprecedented opportuntities for the development of functional membrane systems possessing the combination of robust trace-detection performance and molecular trapping function. These findings not only present clear technological implications, but also provide an improved understanding of the fundamental mechanisms of surface-induced geological and biological processes.

NPG Asia Materials (2016) 8, e245; doi:10.1038/am.2016.15; published online 18 March 2016

\section{INTRODUCTION}

Liquid-solid phase transformation at interfaces is fundamental to many industrial, geological and biological processes. ${ }^{1-11}$ For example, the organization of apatite crystals on the bone surface is closely associated with bone biomineralization. ${ }^{2,6}$ The success in rational control over the nucleation of organic single-crystal on surface allows for obtaining high-resolution structures of proteins or fabricating large arrays of single-crystal filed-effect transistors with unprecedented high performances. ${ }^{8,9}$ In addition, controlling crystallization at interfaces, either for its prevention or for acquiring the desired crystalline properties, is crucial for producing foods with superior quality or with extended shelf life. ${ }^{4}$ However, although a lot have been learned on the process of crystallization, only limited examples of successful manipulation of the crystallization behavior at interfaces are reported. Current strategies to control the kinetics of surface-induced crystallization, including solvents, ${ }^{2}$ organic additives ${ }^{11,12}$ or porous templates, ${ }^{5,7-9}$ have been proposed. For example, it has been well established that rational introduction of water and other organic additives such as collagen, proteoglycans and non-collagenous proteins is an effective way for controlling biomineralization. ${ }^{2}$ Interestingly, the crystallization of molecular liquid from solutions can be engineered by tailoring the nanopore shape of the employed template. ${ }^{5}$ Although these strategies have been proved effective, most of them still derive from the experience acquired from lengthy liquid-phase chemistry approaches.
In the present study, a new surface-directed crystallization mechanism that does not depend on solutions or templates, but is instead driven by the chemical and/or structural inhomogeneity of the substrate, is suggested. This mechanism mainly involves stimulation of inherent potential of interfaces for steering the crystallization kinetics. For this purpose, a new hybrid surface with a unique configuration of 'glass embedded with crystal domain' has been designed, which is in direct contrast with the conventional approach in which the substrate is either in amorphous or in crystalline state (Figure 1). In its simplest form, the heterogeneous surface consists of two phases: a glassy phase embedded with homogeneously distributed crystalline domains (Figure 1c). This unique configuration not only retains the benefit of flat solid substrates for uniform crystallization, but also enables for controlling the size and distribution of the nanocrystalline phase, with spacing down to length scales in the range of the initial stages of crystallization. The resultant nanoscopic alternative structure could yield periodic potential field and could thus be expected to dominate the atomic migration and subsequent crystallization behavior on this unique surface.

\section{MATERIALS AND METHODS}

Design and fabrication of the nanocomposite substrate

A nanocomposite composed of $\mathrm{TiO}_{2}$ nanocrystals homogeneously distributed in a borate glass phase was chosen as the prototypical heterogeneous substrate. The composition of the glass was determined by referring to the phase diagram

\footnotetext{
${ }^{1}$ State Key Laboratory of Luminescent Materials and Devices, Guangdong Engineering Technology Research and Development Center of Special Optical Fiber Materials and Devices, School of Materials Science and Engineering, South China University of Technology, Guangzhou, China; ${ }^{2}$ School of Materials Science and Engineering, Zhejiang University, Hangzhou, China and ${ }^{3}$ Department of Material Chemistry, Graduate School of Engineering, Kyoto University, Kyoto, Japan

Correspondence: Professor S Zhou, State Key Laboratory of Luminescent Materials and Devices, Guangdong Engineering Technology Research and Development Center of Special Optical Fiber Materials and Devices, School of Materials Science and Engineering, South China University of Technology, Wushan Road, Tianhe District, Guangzhou 510640, China.

E-mail: zhoushifeng@scut.edu.cn

Received 3 September 2015; revised 22 November 2015; accepted 3 December 2015
} 

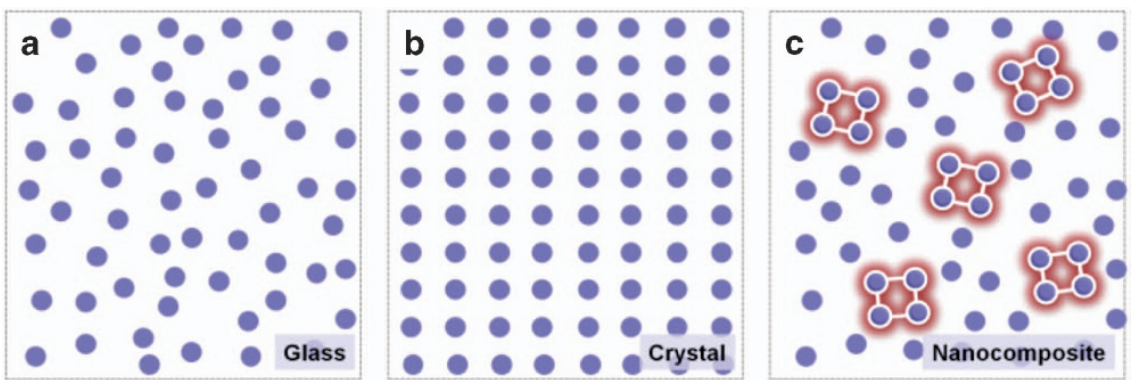

Figure 1 Design of nanocomposite substrate. (a-c) Schematic comparison between homogeneous surface of glass and crystalline substrates and heterogeneous surface of composite substrate.

of $\mathrm{B}_{2} \mathrm{O}_{3} / \mathrm{BaO} / \mathrm{ZnO}$ system and further optimized after numerous trial and error. ${ }^{13}$ The glass had a composition of: $\mathrm{B}_{2} \mathrm{O}_{3} / \mathrm{BaO} / \mathrm{ZnO} / \mathrm{CaO} / \mathrm{Al}_{2} \mathrm{O}_{3} / \mathrm{TiO}_{2}=63$ 19/9/4/1/14 (in mol\%). This composition corresponds to the raw materials used for melting. In a typical synthesis process, analytical pure reagents grade $\mathrm{BaO}$, $\mathrm{ZnO}, \mathrm{CaO}, \mathrm{Al}_{2} \mathrm{O}_{3}, \mathrm{TiO}_{2}$ and boric acid $\left(\mathrm{H}_{3} \mathrm{BO}_{3}\right)$ were used as source materials. Approximately $30 \mathrm{~g}$ batch of the above raw materials was completely mixed and melted in a corundum crucible at $1350^{\circ} \mathrm{C}$ for 30 min under ambient atmosphere. The homogenized melt was quenched onto a preheated stainless brass mold to form glass. Subsequently, the as-made glass was heat-treated at $680{ }^{\circ} \mathrm{C}$ for $2 \mathrm{~h}$ to induce in situ precipitation of $\mathrm{TiO}_{2}$ nanocrystals. Finally, the nanocomposite material obtained was carefully polished and cleaned by sonication in acetone, ethanol and then distilled water. In the comparative test, rutile $\mathrm{TiO}_{2}$ single crystal with an exposed surface orientation of (110) was employed.

\section{Surface-induced crystallization}

$\mathrm{AgNO}_{3}$ was used as precursor for the study of the surface-induced crystallization behaviors of $\mathrm{Ag}_{2} \mathrm{O}$. The substrate was immersed into $10 \mathrm{ml}$ $0.025 \mathrm{~mol} \mathrm{l}^{-1} \mathrm{AgNO}_{3}$ aqueous solution and held in a drying oven at $70{ }^{\circ} \mathrm{C}$ with preservation time from $10 \mathrm{~min}$ to $2 \mathrm{~h}$. Then the substrate was taken out and washed with ethanol twice, followed by drying at room temperature for $2 \mathrm{~h}$.

\section{Characterizations}

The thermal properties of the glass samples were characterized by differential thermal analysis with a heating rate of $10^{\circ} \mathrm{C} \mathrm{min}-1$ in static air conditions. The crystalline phases were identified by X-ray powder diffraction (XRD) using a RIGAKU D/MAX 2550/PC diffractometer with $\mathrm{Cu}$ Ko radiation. Structures and morphology were imaged in a field-emission scanning electron microscope (SEM; JSM6700F, JEOL, Tokyo, Japan). Microstructure analysis was carried out using a JEOL $2010 \mathrm{~F}$ transmission electron microscopy operating at $200 \mathrm{kV}$, equipped with an energy dispersive spectrometer. Trace organic detection was carried out with a confocal Raman spectrometer (Nanofinder30, Tokyo Instruments Co.) in backscattering geometry with a 633-nm laser as excitation source. The laser beam was focused with an objective lens $(\times 40$, numerical aperture $(N A)=0.6)$. The laser power at the sample was maintained at $200 \mu \mathrm{W}$. The acquisition time for spectrum collection was $1 \mathrm{~s}$. Surface-enhanced Raman scattering maps were collected in a region of $100 \times 100 \mu \mathrm{m}^{2}$ with the step size of $2.25 \mu \mathrm{m}^{2}$.

\section{Trace-detection testing}

The porous $\mathrm{Ag}$ membrane was fabricated via gentle photodecomposition of anisotropic $\mathrm{Ag}_{2} \mathrm{O}$ on heterogeneous surface. In the photodecomposition experiment, the substrate was irradiated with ultraviolet light from a $\mathrm{Hg}-\mathrm{Xe}$ lamp with the central wavelength at $\sim 365 \mathrm{~nm}$. The irradiation duration was $6 \mathrm{~h}$. Then, the porous Ag membrane that derived from the as-prepared anisotropic $\mathrm{Ag}_{2} \mathrm{O}$ membrane was carefully transferred to silicon. An aromatic compound, benzenethiol, was employed as the model specimen of tracedetection. The porous Ag membrane was exposed to the benzenethiol $\left(10^{-6} \mathrm{~mol}^{-1}\right)$ solution. Surface-enhanced Raman scattering experiments were conducted with a confocal micro-Raman spectrometer.

\section{Theoretical simulation}

The first-principle calculations were performed using density functional theory from Vienna ab initio simulation package with a plane wave basis. A generalized gradient approximation was used for exchange-correlation function. The core electrons were represented by the projector-augmented-wave potential. Kinetic energy cutoff was set above $400 \mathrm{eV}$. The k-point sampling on the $\mathrm{Ag}_{2} \mathrm{O}$ surface unit cell was $10 \times 10$, with a minimum distance between the neighboring slabs $>20 \AA$, which is large enough to avoid interactions between them. The structure optimization process was performed with force convergence criteria at $0.01 \mathrm{eV} \AA^{-1}$. A theoretical simulation based on the finite difference timedomain method was performed to provide explanation for the origin of the robust performance of the Ag membrane chip. The simulation was performed using a commercially available software (Poynting for Optics; Fujitsu, Kyoto, Japan). In the simulation, two Ag nanoparticles with a radii of $5 \mathrm{~nm}$ and embedded in glass (refractive index $n=1.45$ ) were assumed. The calculations were performed on a cubic grid having a discretization step of $0.2 \mathrm{~nm}$, with perfectly matched layer conditions imposed at the boundaries. To predict the local field enhancement at the nanogap between metal nanoparticles, the differential electric field calculations on the presence or absence of nanoparticles were performed by launching a continuous wave light with a wavelength of $633 \mathrm{~nm}$, and computing its evolution according to the Maxwell's equations. The nanogap width $(d)$ was defined as the shortest distance between the nanoparticles.

\section{RESULTS AND DISCUSSION}

To address the need for the development of an appropriate surface with the designed configuration, we devised a scheme that involves the self-limited nanocrystallization of the glassy phase. ${ }^{14,15}$ The strategy starts with a melt-quenching process for producing a highly homogeneous bulk glass, followed by a thermal activation allowing for in situ precipitation of crystalline domains within the matrix. As the nanocrystallization process in a viscous glassy phase is accompanied by a local enrichment in glass-forming elements around the nanocrystals precipitated, the viscosity at the glass-crystal interface will progressively increase. Consequently, a diffusion barrier will form around these crystals. As a result, a sharp decrease in the crystal-growth velocity can be expected, thus enabling strict control of the crystal size at the nanoscale. Furthermore, the unique interface barrier formed is also crucially important for preventing unfavorable aggregation of nanocrystals, thereby allowing for preserving their spacing, even for high crystallization volume fractions. Based on this scheme, a prototypical nanocomposite composed of $\mathrm{TiO}_{2}$ nanocrystals homogeneously distributed in a borate glass phase was successfully fabricated. The thermal activation condition was guided by the careful differential thermal analysis, Supplementary Figure S1) of meltquenched glasses. The precipitation of $\mathrm{TiO}_{2}$ nanocrystals is evident from the XRD patterns and Raman spectra (Figures 2a and b and Supplementary Figure S2), where the corresponding fingerprint peaks can be clearly observed in the composite sample. Transmission 

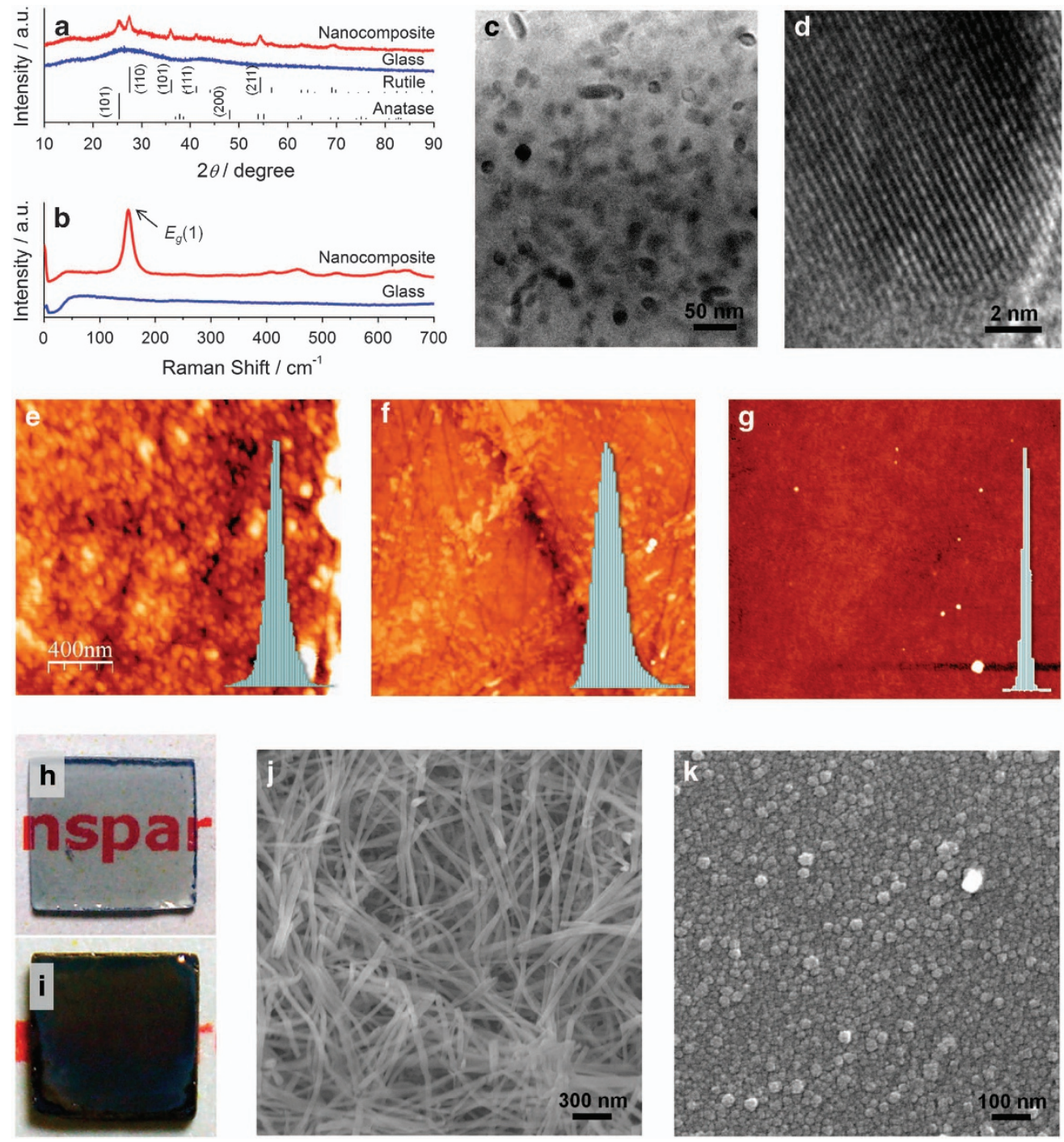

Figure 2 Construction of nanocomposite substrate and crystallization behavior of $\mathrm{Ag}_{2} \mathrm{O}$ on it. (a, b) X-ray powder diffraction (XRD) and Raman patterns of glass and nanocomposite samples, respectively. (c) Typical low-resolution transmission electron microscopy (TEM) micrograph of the nanocomposite. (d) High-resolution TEM image of a single nanocrystal. (e- $\mathbf{g}$ ) Atomic force microscope (AFM) images of nanocomposite (e), glass (f) and TiO ${ }_{2}$ single $\mathrm{crystal}$ (g), respectively. The insets present the roughness analysis and thie surface roughness was estimated to be $\sim 1.65,1.18$ and $0.25 \mathrm{~nm}$, respectively. The scales are same and indicated in e. (h, i) Photographs showing the composite substrate before and after $\mathrm{Ag}_{2} \mathrm{O}$ deposition, respectively. The physical size of composite substrate is $10 \times 10 \times 2 \mathrm{~mm}^{3}$. (j, k) scanning electron microscope (SEM) images of $\mathrm{Ag}_{2} \mathrm{O}$ grown on the composite and glass surface, respectively.

electron microscopy images (Figures $2 \mathrm{c}$ and $\mathrm{d}$ and Supplementary Figure S3) show crystallized $\mathrm{TiO}_{2}$ domains with high density $\left(\sim 5 \times 10^{18} \mathrm{~cm}^{-3}\right)$ and uniform size $(\sim 20 \mathrm{~nm})$ homogeneously embedded in the amorphous phase. Atomic force microscope images of a nanocomposite (Figure 2e), a glass (Figure 2f) and a $\mathrm{TiO}_{2}$ single crystal (Figure $2 \mathrm{~g}$ ) show the marked difference of their surface features, with surface roughness of $\sim 1.65,1.18$ and $0.25 \mathrm{~nm}$, respectively (Supplementary Figure S4). For the nanocomposite (Figure 2e), it can be cleary observed that $\mathrm{TiO}_{2}$ nanocrystals are homogeneously distributed in the glassy phase. A photograph of the composite sample (Figure $2 \mathrm{~h}$ ) shows the excellent transparency of the material, giving further indication of the high dispersibility of the nanocrystals within the glass matrix. These results collaboratively demonstrate the chemical and structural inhomogeneity of the nanocomposite substrate. The effect of the glass composition on the optical quality of the composites was also investigated. It was found that the choice of the composition is crucial for obtaining transparent materials. Transparent composites present the highest interest, as an excellent light transmission over a wide wavelength region is crucial for photonic applications.

$\mathrm{Ag}_{2} \mathrm{O}$ crystallization behavior on the surface of the prepared nanocomposite as described above, composed of a borate glass embedding $\mathrm{TiO}_{2}$ nanocrystals, was investigated in a simple, additivefree $\mathrm{AgNO}_{3}-\mathrm{H}_{2} \mathrm{O}$ binary system. Specifically, the substrate was immersed into the $\mathrm{AgNO}_{3}$ solution $\left(0.025 \mathrm{moll}^{-1}\right)$ and held at a mild temperature $\left(70^{\circ} \mathrm{C}\right)$ for $2 \mathrm{~h}$. Encouragingly, the crystallization of $\mathrm{Ag}_{2} \mathrm{O}$ extended on the entire surface of substrate (Figure $2 \mathrm{i}$ and Supplementary Figure S5). In addition, the SEM examination of the microstructure of the deposited $\mathrm{Ag}_{2} \mathrm{O}$ reveals an unusual one-dimensional (1D) morphology, with a diameter of $\sim 50 \mathrm{~nm}$ and a length/diameter ratio $>200$ (Figure $2 \mathrm{j}$ ). As no $\mathrm{Ag}_{2} \mathrm{O}$ nanoparticle can be observed, the yield of $\mathrm{Ag}_{2} \mathrm{O}$ nanowire is estimated to be close to $100 \%$. In sharp contrast, similar studies carried out on the conventional (non-crystallized) glass and crystal surfaces show that the same 
growth procedure only yields layers of $\mathrm{Ag}_{2} \mathrm{O}$ islands on both of these surfaces (Figure 2k, Supplementary Figures S6 and S7). Furthermore, it must be noted that surface passivation has negligible effects on the anisotropic growth behavior of $\mathrm{Ag}_{2} \mathrm{O}$ on the composite surface (Supplementary Figure S8). In addition, the crystallization of $\mathrm{Ag}_{2} \mathrm{O}$ with a $1 \mathrm{D}$ morphology can also be observed on the surface of composites containing only an anatase phase (Supplementary Figure S9). These initial results strongly indicate that heterogeneoussurface-mediated crystallization control can be achieved based on the proposed mechanism.

To gain better knowledge on the anisotropic growth behavior of $\mathrm{Ag}_{2} \mathrm{O}$ on the heterogeneous surface, the intermediate stages of crystallization were investigated. Several important points were observed.

First, time-resolved SEM images (Figures 3a-g) were obtained from $10 \mathrm{~min}$ to $2 \mathrm{~h}$, and several distinct stages of crystallization could be identified (Figures $3 \mathrm{c}-\mathrm{g}$ ). In the first stage, surface-mediated nucleation resulted in the formation of nanosized aggregates (Figure $3 \mathrm{c}$ ). Subsequently, the second stage of growth begins, which is dominated by the appearance of short nanorods (Figure 3d). These nanorods gradually grow into long $1 \mathrm{D}$ nanowires with a snake-like shape, and ultimately result in the formation of a nanowire membrane (Figures $3 \mathrm{e}-\mathrm{g}$ and Supplementary Figure S10).

Second, high-resolution SEM images at intermediate stages (Figures $3 \mathrm{~h}-1$ ) provided valuable information about the microstructural evolution. The 1D structure formed during the early stage is rather loose, and the aligned particles can even re-decompose into islands (an example is marked with an arrow in Figure 3i). Furthermore, the external particles can easily connect to the aligned part, generating trimers and even more complex tetramer structures (Figures $3 \mathrm{~h}, \mathrm{k}$ and $\mathrm{l}$ ).

Third, comparative studies on the initial crystallization stage of $\mathrm{Ag}_{2} \mathrm{O}$ on both of the homogeneous single crystal and glass surface were conducted (Figure 4). Relatively large $\mathrm{Ag}_{2} \mathrm{O}$ particles were observed as compared to those found on heterogeneous surface
(Figures 4c, $\mathrm{f}$ and i). Furthermore, a closer examination of the microstructure of the base film indicates that the particle islands on the homogeneous single crystal and on the glass surface are relatively dense, which is in sharp contrast to the strikingly loose feature of nanosized aggregates formed on the heterogeneous substrate.

Given the evidences described above, the following model is proposed for describing heterogeneous-surface-mediated crystallization (Figures $4 \mathrm{a}-\mathrm{i}$ ). Once exposed to the metal precursor, the surface of crystalline, glassy materials or even their hybrid state, can support the hydrolysis condensation of metal ions. The tiny crystalline $\mathrm{TiO}_{2}$ domains embedded within the heterogeneous surface can serve as favorable spots for preferential nucleation, which will lead to a decrease in nanoparticle size (Figures $4 \mathrm{~h}$ and i). This strongly contrasts with the scenario observed for crystallization on homogeneous single crystal and glass surfaces, where large-sized $\mathrm{Ag}_{2} \mathrm{O}$ particles are crystallized (Figures 4b, c, e and f). Another intriguing advantage of crystalline-glass alternative nanoscopic structure is the possibility of obtaining an evenly distribited material, eventually inducing the formation of nanogap-rich $\mathrm{Ag}_{2} \mathrm{O}$ nanoislands. This facilitates the creation of numerous hopping sites and can support the orientation of arbitrary grains with enhanced degree of freedom, such as the polar, azimuthal and rotational/twist as shown in Figure 4h. This hypothesis can be confirmed by the XRD characterization which shows that the nanowires grow rapidly with a favorable orientation along $<111>$ (Figure 4j).

To understand the crystallization process, density functional theory calculations of the surface energy were performed (Figures $4 \mathrm{k}-\mathrm{m}$ ). The surface energy of (111), (001) and (110) planes were estimated to be $\sim 1.048,0.993$ and $0.624 \mathrm{eV}$ per surface unit, respectively. The XRD and simulation results collaboratively demonstrate that the anisotropic crystallization trend of $\mathrm{Ag}_{2} \mathrm{O}$ on heterogeneous surface can be boosted: it grows rapidly with favorable orientation along $\langle 111\rangle$ during crystallization for covering the high-energy surface (111). In comparison, no obvious anisotropic crystallization behavior can be observed for both homogenous single-crystal and glass surfaces. Therefore, this
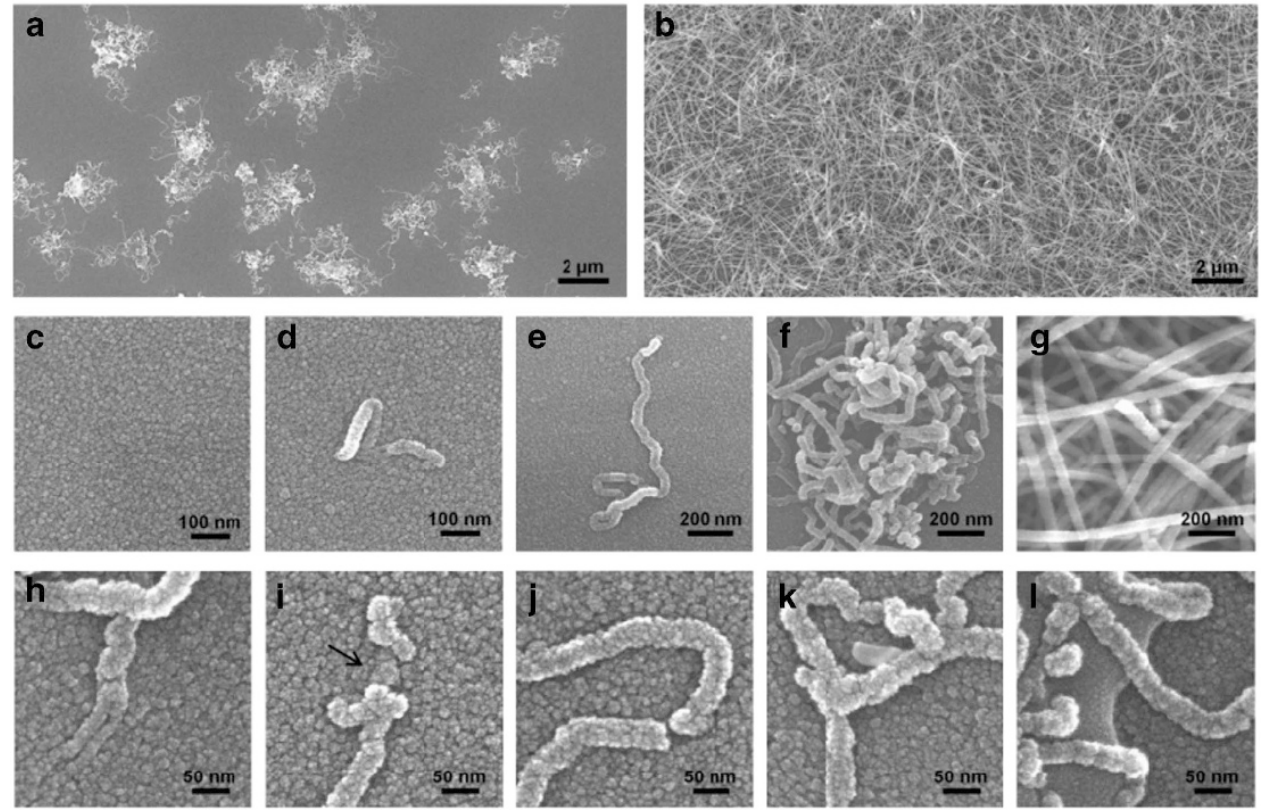

Figure 3 Various stages of the anisotropic growth of $\mathrm{Ag}_{2} \mathrm{O}$ on the heterogeneous surface. (a, b) Typical low-resolution scanning electron microscope (SEM) images taken at the intermediate and the final stages of reaction. (c-g) SEM images of the anisotropic growth of $\mathrm{Ag}_{2} \mathrm{O}$ at various stages for reaction time from 10 min to $2 \mathrm{~h}$. (h-l) High-resolution SEM images of $\mathrm{Ag}_{2} \mathrm{O}$ nanowire obtained at the intermediate stage. 
a

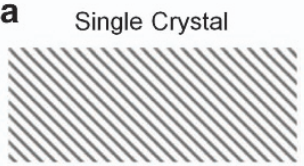

d

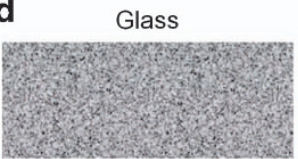

g

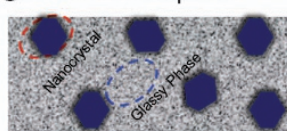

j

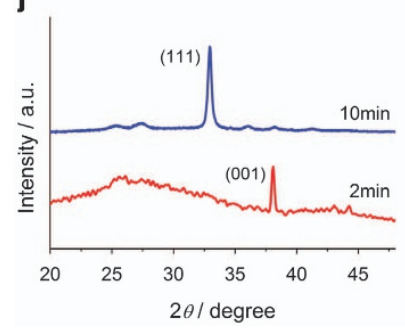

b

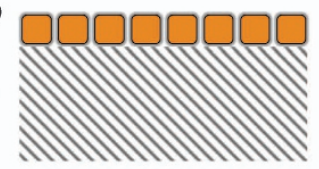

e

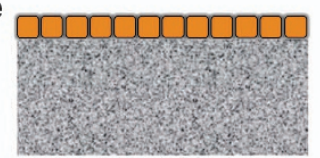

h

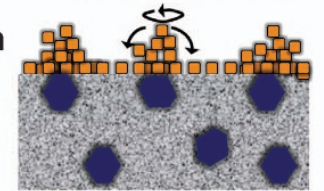

k

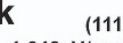

$1.048 \mathrm{eV} /$ surface unit

I

(001)

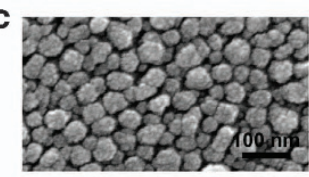

f
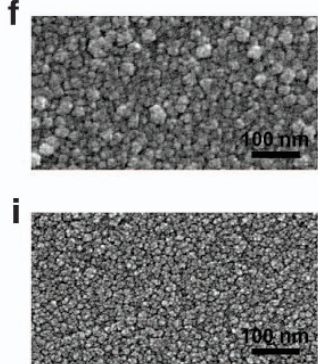

m (110)

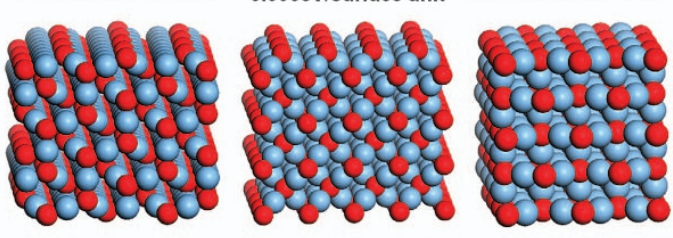

Figure 4 Proposed model of surface-mediated crystallization. (a-i) Schematic showing $\mathrm{Ag}_{2} \mathrm{O}$ crystallization on the surfaces of homogenous single crystal $(\mathbf{a}-\mathbf{c})$, glass ( $\mathbf{d}-\mathbf{f})$ and heterogeneous composite $(\mathbf{g}-\mathbf{i})$. (j) X-ray powder diffraction (XRD) patterns of $\mathrm{Ag}_{2} \mathrm{O}$ on the surfaces of composite. Spectra were recorded at different time points. ( $\mathbf{k}-\mathbf{m})$ Models and calculated surface energies of $\mathrm{Ag}_{2} \mathrm{O}(111),(001)$ and (110) surfaces. $\mathrm{Ag}$ and $\mathrm{O}$ atoms are represented by red and cyan spheres, respectively.

emphasized that the unique $1 \mathrm{D} \mathrm{Ag}_{2} \mathrm{O}$ structure must be a kinetically determined products.

The above-listed findings suggest a mesoscale transformation mechanism from particles to superstructures on the heterogeneous surface. To shed more light on the driving source, further insight is given to the balance of static or transient interparticle forces behind the anisotropic crystallization. The impact of nanoscale forces on crystallization may be qualitatively described by the interaction length scale. Short-range interactions such as van der Waals attraction, which act appreciably only over molecular dimensions, can be excluded since they always lead to undesired aggregation. ${ }^{16}$ The force capable of producing long $1 \mathrm{D} \mathrm{Ag}_{2} \mathrm{O}$ is thus mostly associated with dipolar interactions, as these can have a significant effect over a long-range scale and are surprisingly strong $\left(\sim 10 \mathrm{~kJ} \mathrm{~mol}^{-1}\right) .{ }^{17}$ This fact can be confirmed by the observation of ultra-small aggregations formed on heterogeneous surfaces, which is a strong evidence correlated with the origin of dipole moment. ${ }^{18}$ Specifically, the decrease in aggregate size may help to create significant amount of surface states, resulting in an elevated level of surface charges. ${ }^{18,19}$ Therefore, the tiny dielectric nanoparticles with reduced physical size are more inclined to exhibit noticeable polar character, owing to the strong localization of charges in the dielectric matrix. Furthermore, the distinctive feature of nanogap-rich $\mathrm{Ag}_{2} \mathrm{O}$ nanoislands on heterogeneous surface is highly beneficial for eliminating deleterious screening effect from the neighboring islands, thus enabling the alignment of dipoles through rotation of particles. The results highlight that the intricate interplays between inherent nanoscale force and the unique heterogeneous surface open new possibilities for manipulation of crystallization kinetics.

These encouraging findings prompted investigation into the generality of crystallization control on heterogeneous surfaces. The aqueous chemical growth of various crystalline materials such as transition/rare earth metal-oxides and -hydroxides $\left(\mathrm{Co}(\mathrm{OH})_{2}, \mathrm{ZnO}, \mathrm{Y}\right.$ $(\mathrm{OH})_{3}, \mathrm{Ca}_{3}\left(\mathrm{PO}_{4}\right)_{2}$ and $\left.\mathrm{Fe}(\mathrm{OH})_{3}\right)$ on glass and on heterogeneous surface was examined. A noticeable feature across these material systems is the considerable increase in crystallization efficiency on heterogeneous surface as compared to glass surgace, with the corresponding film thickness roughly twice larger in the case of heterogeneous surface (Supplementary Figure S11a). Another common feature is that the heterogeneous-surface-driven crystallization results in rough films or even diverse superstructures, while relatively flat structures are obtained on glass substrates, as shown in the typical SEM images (Supplementary Figures S11b-k). The morphology of the final products experiences great changes for different material candidates. The physical reason for this difference might be attributable to the specific bonding geometry of a particular system. For example, due to the planar arrangement nature of Co $(\mathrm{OH})_{2}$, it will crystallize into layered structure on either glass or heterogeneous surface. However, the solidification on heterogeneous surface leads to a significant decrease in layer thickness, resulting in the formation of nanosheets (Supplementary Figure S11c). In this context, heterogeneous surface demonstrates the critical role in opening up new pathways toward metastable crystallization

The success in the fabrication of surface-supported superstructures offers unique opportunities for the development of functional membrane systems, thanks to their distinct structural and geometrical features. As an example, it is shown that the thick nanoporous Ag membrane derived from hierarchical $\mathrm{Ag}_{2} \mathrm{O}$ on heterogeneous surface allows for trace-detection with molecular trapping, a function that has been actively pursued in the field of sensors. ${ }^{20-22}$ The porous Ag membrane was fabricated via gentle photodecomposition of anisotropic $\mathrm{Ag}_{2} \mathrm{O}$ on heterogeneous surface, followed by carefully peeling it 

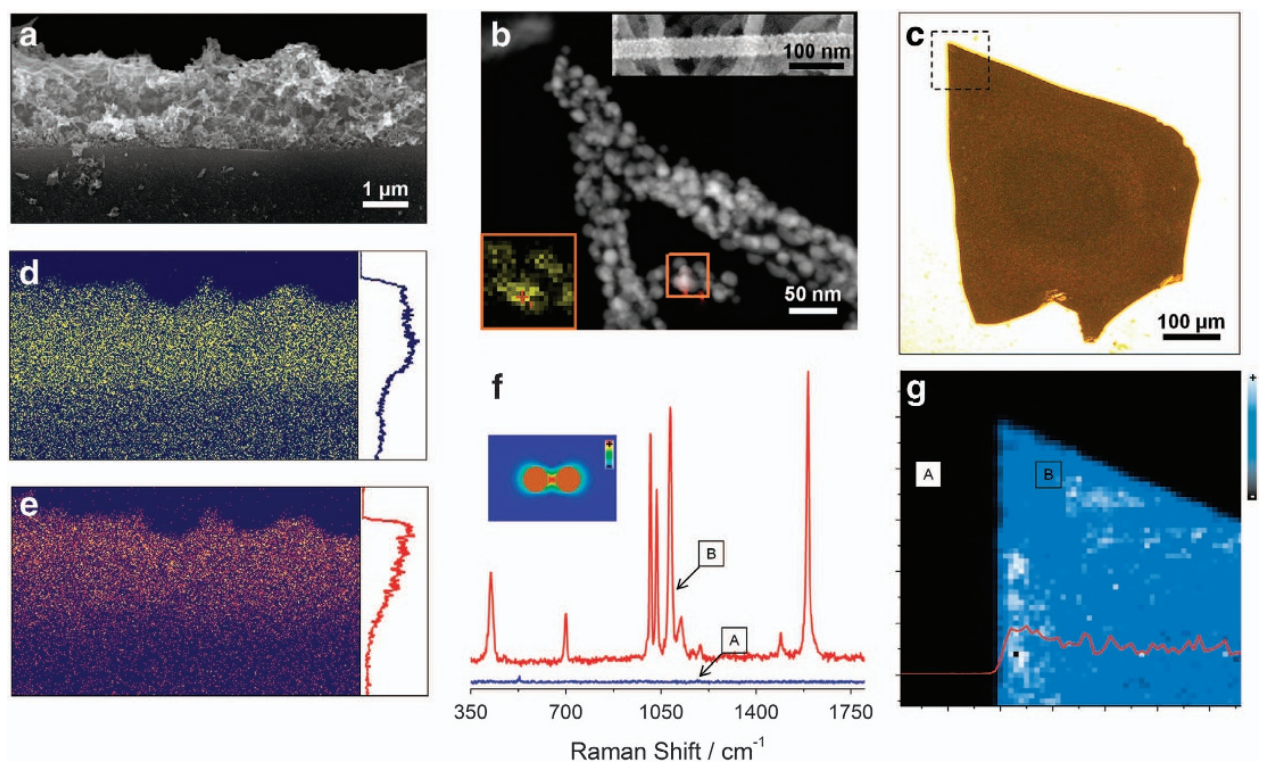

Figure 5 Trace-detection testing of Ag membrane. (a) scanning electron microscope (SEM) image of cross-sectional area of the membrane, showing a porous structure. (b) Transmission electron microscopy (TEM) image of the nanowires. Inset: the corresponding energy-dispersive X-ray (EDS) elemental map Ag (in yellow color) and a SEM image of a single nanowire. (c) Optical image of the nanoporous Ag membrane. (d, e) EDS elemental maps and line profiles of Ag (d) and C (e) in the cross-sectional area of the membrane as shown in a. (f, $\mathbf{g})$ Representative Raman spectra (f) and mapping (g) of $10^{-6} \mathrm{M}$ benzenethiol on nanoporous Ag membrane. Raman spectra measured from the selected area marked in $\mathbf{g}$. Raman mapping measured in a corner of the membrane marked in c by monitoring $\mathrm{C}-\mathrm{C}$ stretching band at $1572 \mathrm{~cm}^{-1}$. The inset in $\mathbf{f}$ is the calculated spatial map of the optical near-field intensity for Ag islands with a 2.5$\mathrm{nm}$ wide gap. The inset in $\mathbf{g}$ shows the line profile of Raman intensity.

off from the substrate and transferring it to the surface of a silicon substrate. The SEM imaging (Figure 5a) shows the cross-sectional view of the membrane $(\sim 2.5 \mu \mathrm{m}$ thick), which consists of nanowires. A combination of SEM and transmission electron microscopy (Figure $5 \mathrm{~b}$ ) studies reveals the formation of both inter-connected mesopores $(\sim 5 \mathrm{~nm})$ and macropores $(\sim 500 \mathrm{~nm})$, potentially rendering the materials with molecular trapping attribute. To investigate it, the membrane was exposed to an aromatic compound, and the carbon distribution was analyzed. As shown in Figures 5e, a gradient distribution of the carbon along the Ag membrane (Figure 5d) can be obsered, firmly confirming the successful trapping of the aromatic molecules. The possibility that the $\mathrm{C}$ originates from other contaminations can be ruled out based on the comparison experiment (Supplementary Figure S12). Trapability of the porous membrane to be used as an ultrasensitive trace-detection chip was further tested by employing benzenethiol as a model specimen. Micro-Raman microscopy was used to identify trace organic molecules. Figure $5 f$ shows the typical micro-Raman spectra recorded at spot A (on silicon) and B (on chip) after exposure to trace benzenethiol with extremely low concentration $\left(10^{-6} \mathrm{M}\right)$. Encouragingly, the fingerprint bands of benzenethiol at $420 \mathrm{~cm}^{-1}(\nu 7 \mathrm{a}, \nu \mathrm{C}-\mathrm{S}), 700 \mathrm{~cm}^{-1}(\nu 6 \mathrm{a}, \nu \mathrm{C}-\mathrm{S})$, $1010 \mathrm{~cm}^{-1}$ ( $\left.\nu 12\right), 1033 \mathrm{~cm}^{-1}(\nu 18 \mathrm{a}), 1082 \mathrm{~cm}^{-1}(\mathrm{\nu 1})$ and $1572 \mathrm{~cm}^{-1}$ ( $\omega 8 \mathrm{a}$ and $\omega 8 \mathrm{~b}, \omega \mathrm{C}=\mathrm{C}$ ) can be clearly distinguished on the nanoporous Ag membrane chip; ${ }^{23}$ This is in stark contrast with the scenario observed on the silicon substrate, where the characteristic bands are entirely unidentifiable. The signal collected from the membrane chip is also significantly stronger $(\sim$ sevenfold $)$ than that from the standard $\mathrm{Ag}$ aggregation widely used in commercial detection system (Supplementary Figure S13). ${ }^{24}$ Raman mapping of the signal intensity and drift of the characteristic band at $1572 \mathrm{~cm}^{-1}$ (C-C stretching) in the corner region $(100 \times 100 \mu \mathrm{m})$ of the membrane was also carried out (Figure 5g and Supplementary Figure S14). The results clearly demonstrate that the membrane chip provides an efficient and portable trace-detection platform with sufficiently homogeneous signal and negligible drift noise $\left(<15 \mathrm{~cm}^{-1}\right)$ over the whole surface, which is of critical importance for the fabrication of reliable integrated sensors. ${ }^{25,26}$

The origin of the robust performance of the membrane chip is interpreted as being twofold. On one hand, the unique mesoporous structure composed of islands with nanogap spacing $(<10 \mathrm{~nm})$ can be expected to generate strongly localized and highly dense 'hot spots' for the local field enhancement. This can be supported by theoretical simulations based on the finite difference time-domain method. The nanogap-width-dependent differential electric field in the nanogap region clearly shows a noticeable local field enhancement at the nanogap with a width of several nanometers (Supplementary Figure S15). Furthermore, the calculated spatial map of the optical near-field intensity for the pairs of $\mathrm{Ag}$ nanoparticles with various nanogap width of $0,1,2.5,5,7.5$ and $10 \mathrm{~nm}$ indicates that the local field intensity exhibits a sharp maximum at the strongly coupled necks connecting particles (Figure 5f, inset and Supplementary Figure S15). This is believed to greatly facilitate the amplification of vibrational or rotational signal resulting from the inelastic scattering process between a photon and a molecule located at the neck region. ${ }^{27,28}$

On the other hand, the porous network combines the advantages of enormous surface area and excellent capillary action, thus not only allowing for trapping of analytes but also ensuring their full access to the 'hot spots', as well as analyte retention. It can also be noted that, in addition to the trace-detection application demonstrated above, the constructed nanoporous membrane is also promising to serve other purposes such as catalyst supports, nanoelectronic scaffolds, filters for biomolecule purification and switchable fluid/ionic transport. ${ }^{29-31}$ Furthermore, unique membranes with porous architectures may potentially show superior electrochemical properties. ${ }^{32}$ The success in simultaneously tailoring the crystallization efficiency and the morphology of the products on the heterogeneous surface is also of 
special interest for the control of both bone and tooth formation. ${ }^{33}$ The new findings presented in this study also provide an improved understanding of fundamental mechanism of surface-induced mineral deposition. Indeed, most of the previous studies on biomineralization are exclusively focused on homogeneous substrates. The heterogeneous surfaces described here are considered to be much closer to the complex natural systems. These finding thus also give valuable clues on the origin of diverse metastable biomineral products frequently observed in nature. ${ }^{34,35}$

\section{CONCLUSIONS}

In this study, a new surface-directed crystallization mechanism enabled by the chemical and structural inhomogeneity of the substrate has been proposed and demonstrated. As a proof-of-concept demonstration, the success in steering the crystallization kinetics has been achieved on designed surfaces with a 'glass embedded with crystal domain' configuration. Benefiting from the intricate interplays between nanoscale forces and heterogeneous surface, the strategy offers new opportunities in the large-scale development of various metastable crystallization products such as nanowire and nanoporous membrane (Figures 4 and 5). The proposed approach also serves practical purposes. For example, the obtained Ag membrane was demonstated to show robust label-free trace-detection performance with molecular trapping function that has been actively pursued in the field of sensors.

\section{CONFLICT OF INTEREST}

The authors declare no conflict of interest.

\section{ACKNOWLEDGEMENTS}

We gratefully acknowledge the financial support from the National Natural Science Foundation of China (Grant 11474102), Chinese Program for New Century Excellent Talents in University (Grant NCET-13-0221), Guangdong Natural Science Funds for Distinguished Young Scholar (Grant S2013050014549), Fundamental Research Funds for the Central University and the Scientific Research Foundation for Returned Overseas Chinese Scholars, State Education Ministry.

1 Mullin, J. W. Crystallization 4th edn (Butterworth-Heinemann, Germany, 2001).

2 Wang, Y., Euw, S. V., Fernandes, F. M., Cassaignon, S., Selmane, M., Laurent, G., Pehau-Arnaudet, G., Coelho, C., Bonhomme-Coury, L., Giraud-Guille, M. M., Babonneau, F., Azaïs, T. \& Nassif, N. Water-mediated structuring of bone apatite. Nat. Mater. 12, 1144-1153 (2013).

3 Treat, N. D., Malik, J. A. N., Reid, O., Yu, L., Shuttle, C. G., Rumbles, G., Hawker, C. J. Chabinyc, M. L., Smith, P. \& Stingelin, N. Microstructure formation in molecular and polymer semiconductors assisted by nucleation agents. Nat. Mater. 12, 628-633 (2013).

4 Hartel, R. W. Advances in food crystallization. Annu. Rev. Food Sci. Technol. 4, 277-292 (2013).

5 Diao, Y., Harada, T., Myerson, A. S., Hatton, T. A. \& Trout, B. L. The role of nanopore shape in surface-induced crystallization. Nat. Mater. 10, 867-871 (2011).

6 Dey, A., Bomans, P. H. H. F., Müller, A., Will, J., Frederik, P. M., de With, G. \& Sommerdijk, N. A. The role of prenucleation clusters in surface-induced calcium phosphate crystallization. Nat. Mater. 9, 1010-1014 (2010).

7 Hamilton, B. D., Weissbuch, I., Lahav, M., Hillmyer, M. A. \& Ward, M. D. Manipulating crystal orientation in nanoscale cylindrical pores by stereochemical inhibition. J. Am. Chem. Soc. 131, 2588-2596 (2009).

8 Briseno, A. L., Mannsfeld, S. C. B., Ling, M. M., Liu, S., Tseng, R. J., Reese, C., Roberts, M. E., Yang, Y., Wudl, F. \& Bao, Z. Patterning organic single-crystal transistor arrays. Nature 444, 913-917 (2006).

9 Chayen, N. E., Saridakis, E. \& Sear, R. P. Experiment and theory for heterogeneous nucleation of protein crystals in a porous medium. Proc. Natl Acad. Sci. USA 103, 597-601 (2006).

10 Cacciuto, A., Auer, S. \& Frenkel, D. Onset of heterogeneous crystal nucleation in colloidal suspensions. Nature 428, 404-406 (2004).
$11 \mathrm{He}, \mathrm{G} .$, Dahl, T., Veis, A. \& George, A. Nucleation of apatite crystals in vitro by selfassembled dentin matrix protein 1. Nat. Mater. 2, 552-558 (2003).

12 Wang, Y., Azaïs, T., Robin, M., Vallée, A., Catania, C., Legriel, P., Pehau-Arnaudet, G. Babonneau, F., Giraud-Guille, M. M. \& Nassif, N. The predominant role of collagen in the nucleation, growth, structure and orientation of bone apatite. Nat. Mater. 11, 724-733 (2012).

13 Masai, H., Toda, T., Takahashi, Y. \& Fujiwara, T. Fabrication of anatase precipitated glass-ceramics possessing high transparency. Appl. Phys. Lett. 94, 151910 (2009).

14 Bhattacharyya, S., Bocker, C., Heil, T., Jinschek, J. R., Höche, T., Rüssel, C. \& Kohl, H. Experimental evidence of self-limited growth of nanocrystals in glass. Nano Lett. 9 2493-2496 (2009).

15 Zhou, S., Li, C., Yang, G., Bi, G., Xu, B., Hong, Z., Miura, K., Hirao, K. \& Qiu, J. Selflimited nanocrystallization-mediated activation of semiconductor nanocrystal in an amorphous solid. Adv. Funct. Mater. 23, 5436-5443 (2013).

16 Bishop, K. J. M., Wilmer, C. E., Soh, S. \& Grzybowski, B. A. Nanoscale forces and their uses in self-assembly. Small 5, 1600-1630 (2009).

17 Tang, Z., Kotov, N. A. \& Giersig, M. Spontaneous organization of single CdTe nanoparticles into luminescent nanowires. Science 297, 237-240 (2002).

18 Shim, M. \& Guyot-Sionnest, P. Permanent dipole moment and charges in colloidal semiconductor quantum dots. J. Chem. Phys. 111, 6955-6964 (1999).

19 Avraam, P. W., Hine, N. D. M., Tangney, P. \& Haynes, P. D. Factors influencing the distribution of charge in polar nanocrystals. Phys. Rev. B 83, 241402 (2011).

20 Álvarez-Puebla, R. A., Contreras-Cáceres, R., Pastoriza-Santos, I., Pérez-Juste, J. \& Liz-Marzán, L. M. Au@pNIPAM colloids as molecular traps for surface-enhanced, spectroscopic, ultra-sensitive analysis. Angew. Chem. Int. Ed. 48, 138-143 (2009).

21 Álvarez-Puebla, R. A. \& Liz-Marzán, L. M. Traps and cages for universal SERS detection. Chem. Soc. Rev. 41, 43-51 (2012).

22 Schlücker, S. Surface-enhanced raman spectroscopy: concepts and chemical applications. Angew. Chem. Int. Ed. 53, 4756-4795 (2014).

23 Zhou, S., Matsuoka, T., Shimotsuma, Y., Sakakura, M., Nishi, M., Hong, Z., Qiu, J., Hirao, K. \& Miura, K. Localized control of light-matter interactions by using nanoscale asymmetric $\mathrm{TiO}_{2}$. Nanotechnology 23, 465704 (2012).

24 Amezcua-Correa, A., Yang, J., Finlayson, C. E., Peacock, A. C., Hayes, J. R., Sazio, P. J. A., Baumberg, J. J. \& Howdle, S. M. Surface-enhanced raman scattering using microstructured optical fiber substrates. Adv. Funct. Mater. 17, 2024-2030 (2007).

25 Abalde-Cela, S., Ho, S., Rodríguez-González, B., Correa-Duarte, M. A. Álvarez-Puebla, R. A., Liz-Marzán, L. M. \& Kotov, N. A. Loading of exponentially grown $\mathrm{LBL}$ films with silver nanoparticles and their application to generalized SERS detection. Angew. Chem. Int. Ed. 48, 5326-5329 (2009).

26 Oh, Y. \& Jeong, K. Glass nanopillar arrays with nanogap-rich silver nanoislands for highly intense surface enhanced raman scattering. Adv. Mater. 24, 2234-2237 (2012).

27 Maier, S. A. Plasmonics: Fundamentals and Applications (Springer, New York, NY, USA, 2007).

28 Chirumamilla, M., Toma, A., Gopalakrishnan, A., Das, G., Zaccaria, R. P., Krahne, R., Rondanina, E., Leoncini, M., Liberale, C., De Angelis, F. \& Di Fabrizio, E. 3D nanostar dimers with a sub-10-nm gap for single-/few-molecule surface-enhanced raman scattering. Adv. Mater. 26, 2353-2358 (2014).

29 Yuan, J., Liu, X., Akbulut, O., Hu, J., Suib, S. L., Kong, J. \& Stellacci, F. Supenwetting nanowire membranes for selective absorption. Nat. Nanotechnol. 3, 332-336 (2008).

30 Gao, H., Xu, L., Long, F., Pan, Z., Du, Y., Lu, Y., Ge, J. \& Yu, S. H. Macroscopic freestanding hierarchical $3 D$ architectures assembled from silver nanowires by ice templating. Angew. Chem. Int. Ed. 53, 4561-4566 (2014).

31 Xue, Y., Markmann, J., Duan, H., Weissmüller, J. \& Huber, P. Switchable imbibition in nanoporous gold. Nat. Commun. 5, 4237 (2014).

32 Wang, L., Lin, C., Zhang, F. \& Jin, J. Phase transformation guided single-layer $\beta-\mathrm{Co}(\mathrm{OH}) 2$ nanosheets for pseudocapacitive electrodes. ACS Nano 8 , 3724-3734 (2014).

33 Stephen, M. Biomineralization: principles and concepts in bioinorganic materials and chemistry (Oxford University Press, New York, NY, USA, 2001).

34 Brown, W. E. \& Chow, L. C. Chemical properties of bone mineral. Annu. Rev. Mater. Sci. 6, 213-236 (1976).

35 Olszta, M. J., Cheng, X., Jee, S. S., Kumar, R., Kim, Y., Kaufman, M. J., Douglas, E. P. \& Gower, L. B. Bone structure and formation: a new perspective. Mater. Sci. Eng. R $\mathbf{5 8}$ 77-116 (2007).

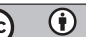

This work is licensed under a Creative Commons Attribution 4.0 International License. The images or other third party material in this article are included in the article's Creative Commons license, unless indicated otherwise in the credit line; if the material is not included under the Creative Commons license, users will need to obtain permission from the license holder to reproduce the material. To view a copy of this license, visit http:// creativecommons.org/licenses/by/4.0/ 